\title{
2-D spectroscopy and modeling of the biconical ionized gas in NGC 4388*
}

\author{
S. Ciroi ${ }^{1}$, M. Contini ${ }^{1,2}$, P. Rafanelli ${ }^{1}$, and G. M. Richter ${ }^{3}$ \\ 1 Dipartimento di Astronomia, Vicolo dell'Osservatorio 2, 35122 Padova, Italy \\ e-mail: piraf@pd.astro.it \\ 2 School of Physics and Astronomy, Tel-Aviv University, Ramat-Aviv, Tel-Aviv, 69978 Israel \\ e-mail: contini@post.tau.ac.il \\ 3 Astrophysikalisches Institut Potsdam, An der Sternwarte 16, 14482 Potsdam, Germany \\ e-mail: gmrichter@aip.de
}

Received 9 September 2002 / Accepted 23 December 2002

\begin{abstract}
We present recent results from spectroscopic data and modeling of the biconical ionized gas in the Seyfert-2 galaxy NGC 4388. A field of $\sim 2.6 \times 2.4 \mathrm{kpc}$ centered on the nucleus has been observed by means of the modern technique of integral field spectroscopy. The analysis of more than two hundred spectra allowed to study the physical characteristics of the gas in the surroundings of the active nucleus. The South-West ionization cone, revealed by the [O III] $\lambda 5007 / \mathrm{H} \beta$ excitation map, shows high emission line ratios not completely supported by simple photoionization. Composite models which account for the combined effects of photoionization and shock show that such high $\left[\mathrm{O} \mathrm{IIII]/H} \beta\right.$ line ratios are emitted by low density $\left(n_{0}=\right.$ $\left.30 \mathrm{~cm}^{-3}\right)$ gas inside large $(D>1 \mathrm{pc})$ shocked clouds $\left(V_{\mathrm{s}}=100 \mathrm{~km} \mathrm{~s}^{-1}\right)$ reached by a relatively low flux from the active nucleus. The data of the VEELR in the North-East cone by Yoshida et al. (2002) have been modeled. The results confirm that photoionization is the prevailing mechanism, but nontheless weak shocks are under way between colliding clouds with small $(<1 \mathrm{pc})$ sizes and low densities $n_{0} \leq 100 \mathrm{~cm}^{-3}$, moving outward at relatively low velocities $\left(V_{\mathrm{s}}=100 \mathrm{~km} \mathrm{~s}^{-1}\right)$.
\end{abstract}

Key words. techniques: spectroscopic - galaxies: nuclei - galaxies: Seyfert - shock waves - galaxies: individual: NGC 4388

\section{Introduction}

The prevailing picture of an AGN structure, the so-called Unified Model, provides for a central supermassive BlackHole $(\mathrm{BH})$, whose gravitational potential energy is the ultimate source of the AGN luminosity. Matter falling into the BH loses angular momentum through viscous and turbulent processes in an accretion disk, resulting into emission of photons in the ultraviolet, soft-X and hard-X ray wavelength domains (Urry \& Padovani 1995).

According to the Unified Model the central source is surrounded by a dense obscuring thick torus which is able to restrict the emergent radiation from the nucleus to a bipolar cone with an opening angle determined by the torus geometry (Pogge 1989). Imaging in optical emission lines shows in some cases high ionization regions with a conical and/or biconical morphology. These so-called ionization cones are direct evidence that radiation escapes anisotropically (Mulchaey et al. 1994).

In this frame we have carried out an observational campaign to study the circumnuclear and extranuclear environment of a spectroscopically selected sample of Seyfert

Send offprint requests to: S. Ciroi, e-mail: ciroi@pd.astro.it

* Based on observations at SAO 6-m telescope (Russia). galaxies (Rafanelli et al. 1995) by means of the integral field spectroscopy. This technique offers much more advantages than the traditional long-slit spectroscopy, because it allows us to observe extended regions of nearby galaxies providing simultaneously recorded spectral information $(\lambda)$ along two spatial directions ( $\alpha$ and $\delta$ ).

Here we present results from the observations of the Seyfert-2 galaxy NGC 4388 , a nearby $(z=0.0084)$ highly inclined spiral galaxy $\left(i \sim 75^{\circ}\right)$, believed located in the core of the Virgo cluster. Indeed observed distortions in the outermost optical isophotes are a clue of the possible interaction between this galaxy and the other members of the cluster.

The IUE spectra of NGC 4388 revealed the presence of an UV source occulted from direct view and producing an ionizing continuum with a steep spectral slope (Ferland \& Osterbrock 1986; Kinney et al. 1991; Kinney et al. 1993).

Narrow-band $\mathrm{H} \alpha+[\mathrm{N} \mathrm{II}]$ and [O III] $\lambda 5007$ images pointed out the presence of emission line regions with highly ionized gas distributed into two opposite cones: a S-W cone extended to $30^{\prime \prime}$ and a N-E plume extended up to $50^{\prime \prime}$ above the galactic disk. Spectroscopic analysis confirmed the photoionization by the central power-law continuum as the principal excitation mechanism (Pogge 1988; Petitjean \& Durret 1993). 


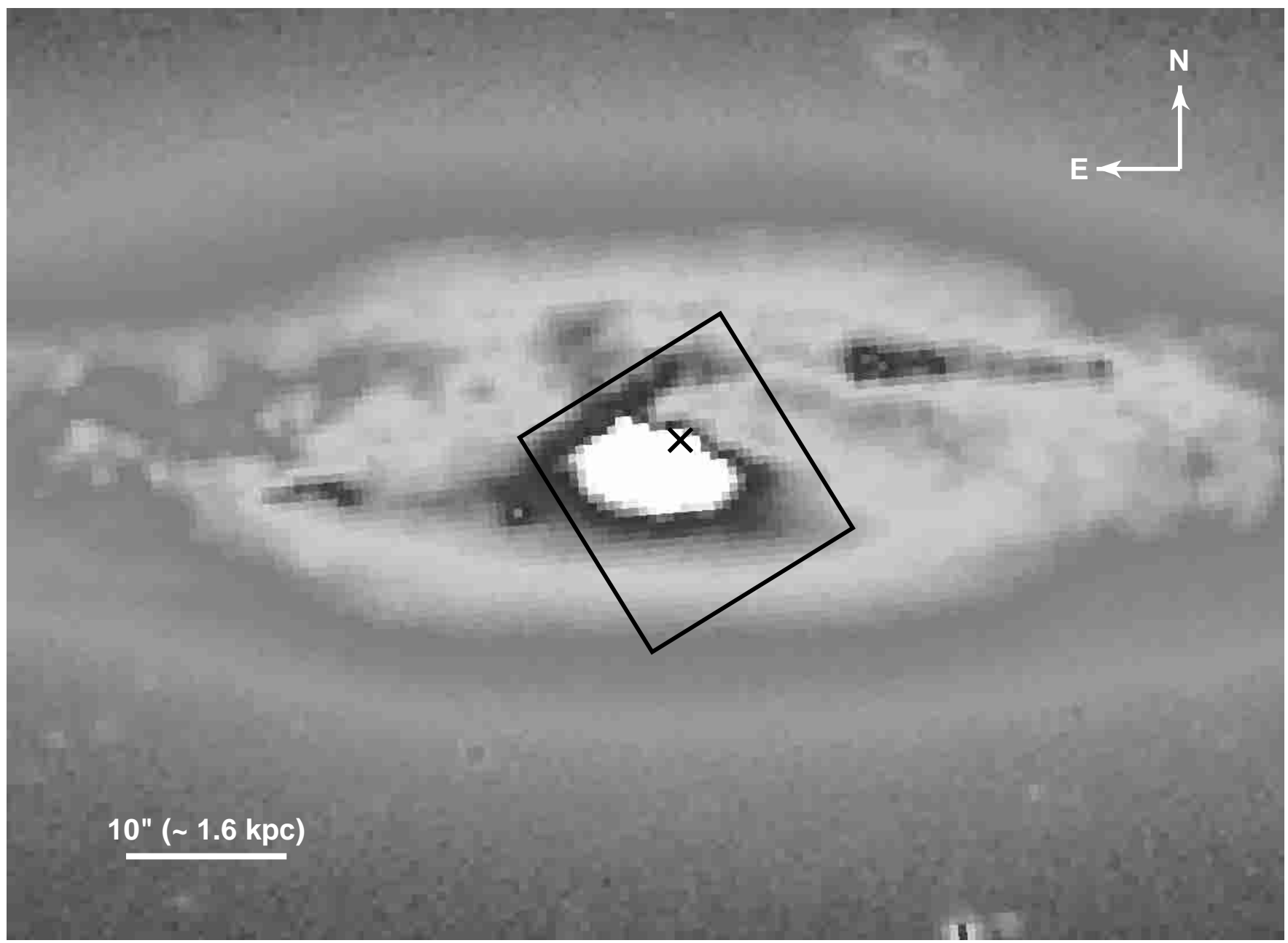

Fig. 1. The position of the spectrograph over the galaxy. The $\times$ symbol indicates the assumed position of the central engine.

Kinematic data obtained with Fabry-Perot Interferometer (Veilleux et al. 1999b) revealed non-rotational blueshifted velocities in the extraplanar gas northeast of the nucleus, likely produced by a bipolar outflow.

Using deep narrow-band imaging obtained with the Suprime-Cam mounted at the Subaru Telescope, Yoshida et al. (2002; hereafter YOS02) have recently found a very large emission line region (VEELR) extended up to $\sim 35 \mathrm{kpc}$ northeast of the nucleus. It consists of many gas clouds or filaments, part of which (within $12 \mathrm{kpc}$ ) are clearly ionized by the nuclear radiation. They claim that the most plausible origin of this ionized gas is tidal debris due to a past interaction with a gas-rich dwarf galaxy.

Moreover at radio wavelengths NGC 4388 shows a two sided, asymmetric structure with a flat spectrum central component, offset by $\Delta \alpha=1.2^{\prime \prime}$ and $\Delta \delta=2.6^{\prime \prime}$ from the apparent optical nucleus, a diffuse blob to the North and an elongated feature to the South with a compact blob in it (Hummel \& Saikia 1991). Several radio maps have shown that this feature is clearly associated with the S-W ionization cone, suggesting the possibility of an interaction between the radio jet and clouds of the ionized gas (Falcke et al. 1998). Such interaction generates shock fronts, which cannot be neglected in the interpretation of the spectra.
In this paper we investigate the physical conditions in the extended biconical ionized gas of NGC 4388 through the modeling of the optical emission line and continuum spectra observed in different regions. Both the ionizing radiation from the active nucleus and the shock effects are consistently accounted for. In Sect. 2 the observations and modeling of the S-W cone are presented. In Sect. 3 the N-E cone is considered and the VEELR is modeled on the basis of YOS02 data. The continuum SED is discussed in Sect. 4. Concluding remarks follow in Sect. 5.

\section{The South-West cone}

\subsection{Observations and data reduction}

NGC 4388 was observed in March 1998 at the 6-m telescope of the Special Astrophysical Observatory (Russia) with the MultiPupil Fiber Spectrograph (MPFS), an integral field unit made by an array of $16 \times 15$ microlenses coupled with a closepacked bundle of optical fibers, which carry the incoming signal to the spectrograph. Each of the 240 elements was covering a region of $1^{\prime \prime} \times 1^{\prime \prime}$ corresponding to a total field of view of about $2.6 \times 2.4 \mathrm{kpc}$ (assuming $H_{0}=75 \mathrm{~km} \mathrm{~s}^{-1} \mathrm{Mpc}^{-1}$ ). Two $600 \mathrm{~s}$ exposures were taken using a TK-1024 CCD, which has 
a pixel size of $24 \mu \mathrm{m}$, and orienting the largest side of the array at $\mathrm{PA} \sim 34^{\circ}$ (Fig. 1). The grating was chosen to give a spectral range of $\sim 4400-7100 \AA$, with a resolution of $10 \AA$ and a dispersion of $5.3 \AA / \mathrm{px}$.

The reduction of the data was carried out by means of a software package, developed within our group, and running under IRAF ${ }^{1}$ environment.

After the usual steps of bias and dark subtraction, all the frames were cross-correlated, in order to compensate for the shifts between them caused by mechanical flexions of the instrument. Then a flat field was chosen as reference image to obtain the geometrical scheme of the spectra arrangement: the position, width and layout along the dispersion direction of every spectrum stored in it were defined and the resulting scheme was subsequently applied to all the images in order to extract for each of them the 2401 -D spectra. The dispersion solution for wavelength calibration was determined by fitting the line positions of He-Ne-Ar lamp exposures with low order Chebyshev polynomial functions. The sky subtraction was performed by averaging the output of 8 fibers devoted to the night sky observation and placed at a distance of $4.5^{\prime}$ from the center of the field of view. Then the data were flux calibrated by observing the spectrophotometric standard star HZ 44: all its flat-fielded, wavelength calibrated and sky subtracted spectra were summed together in order to collect the total incoming flux, to obtain the function necessary to convert counts in physical units and to account for the sensitivity response of the system at different wavelengths. Finally, cosmic rays were removed by combining corresponding spectra of the two exposures of NGC 4388 and a correction for Galactic extinction $\left(A_{V}=0.08\right)$ was applied. Figure 2 shows some examples of the reduced spectra chosen with different signal-to-noise ratio in the field of view.

The positions, widths and fluxes of all the emission lines detectable $3 \sigma$ over the continuum were measured using the IRAF task SPLOT. In case of close blending like $\mathrm{H} \alpha+$ [N II] $\lambda \lambda 6548,6583$ or [S II] $\lambda \lambda 6717,6731$, better results in performing an interactive multiGaussian fit of the total profile could be obtained by using the MIDAS package ALICE, whose input parameters (a first estimate of amplitude, center and sigma of the fitting Gaussian) are independently fixable.

\subsection{Data analysis}

In order to get accurate estimate of line fluxes and ratios it is necessary to take into account that emission lines in galaxies are generally affected, and in some cases even dominated, by the underlying starlight contribution. It is possibile to remove the stellar features by subtracting an absorption-line "template" galaxy spectrum built by means of spectral synthesis techniques (see e.g. Bonatto et al. 1989), or directly obtained from the observation of early-type spirals. In our case the second approach was used, chosing a spectrum of a S0 galaxy extracted from our database which best matched the stellar

${ }^{1}$ IRAF is distributed by the National Optical Astronomy Observatories, which are operated by the Association of Universities for Research in Astronomy, Inc., under cooperative agreement with the National Science Foundation

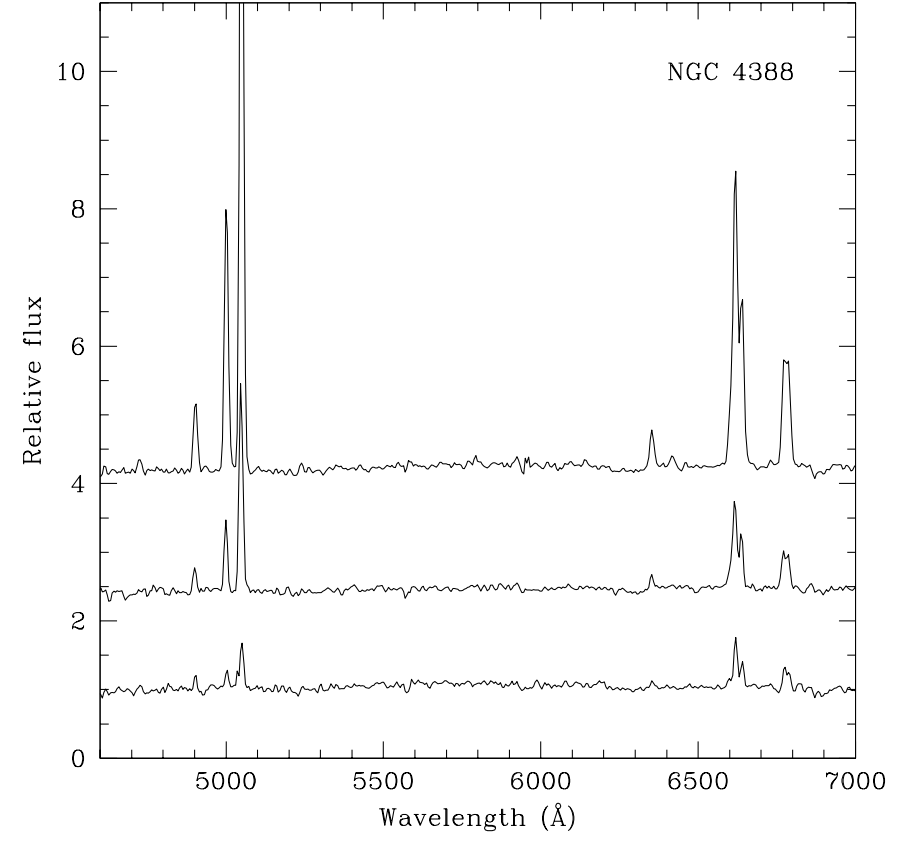

Fig. 2. Examples of three 1-D spectra after the reduction. They are representative of different signal-to-noise ratio in the field of view.

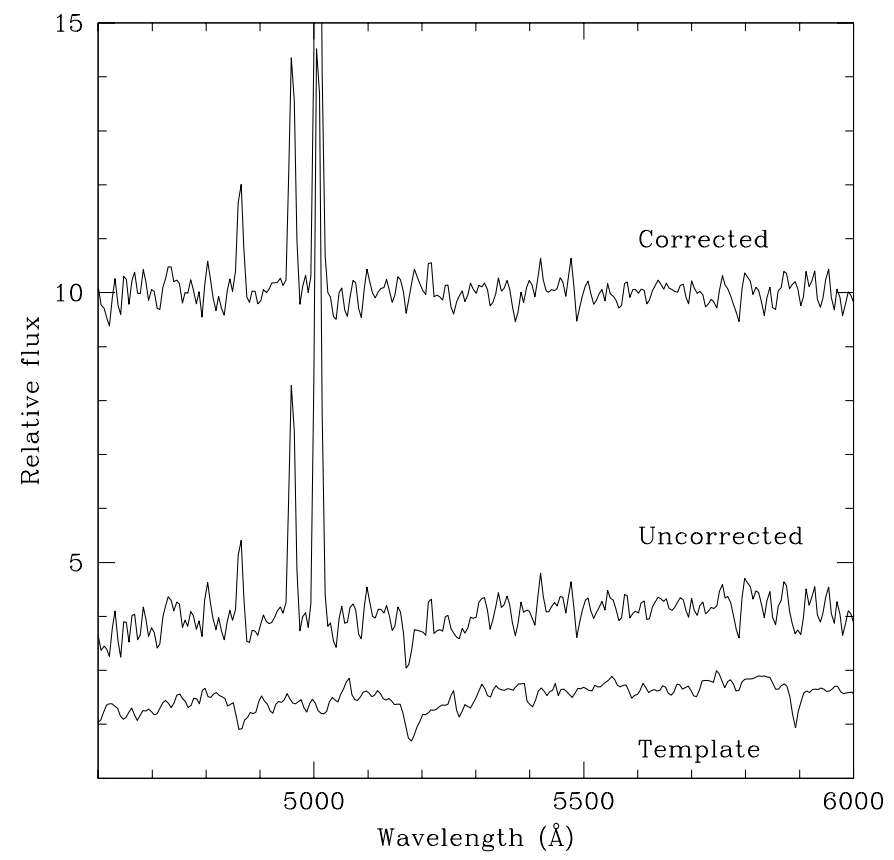

Fig. 3. Example of the underlying starlight correction. An absorptionline "template" spectrum, conveniently rescaled, is subtracted from one of the 240 observed spectra in NGC 4388. The result shows that metallic lines are canceled out and the underlying $\mathrm{H} \beta$ absorption is removed.

features of most of NGC 4388 spectra, and following the suggestions given by Ho et al. (1993). As a whole, the resulting correction was considered satisfactory (an example is given in Fig. 3). The metallic lines (Mg I $\lambda 5175$, Fe I $\lambda 5269$ and $\mathrm{Na}$ I 15892 ) were almost always canceled out after the subtraction. Only in some cases the stellar features of the template have been strengthened to better match the $\mathrm{H} \beta$ absorption, 


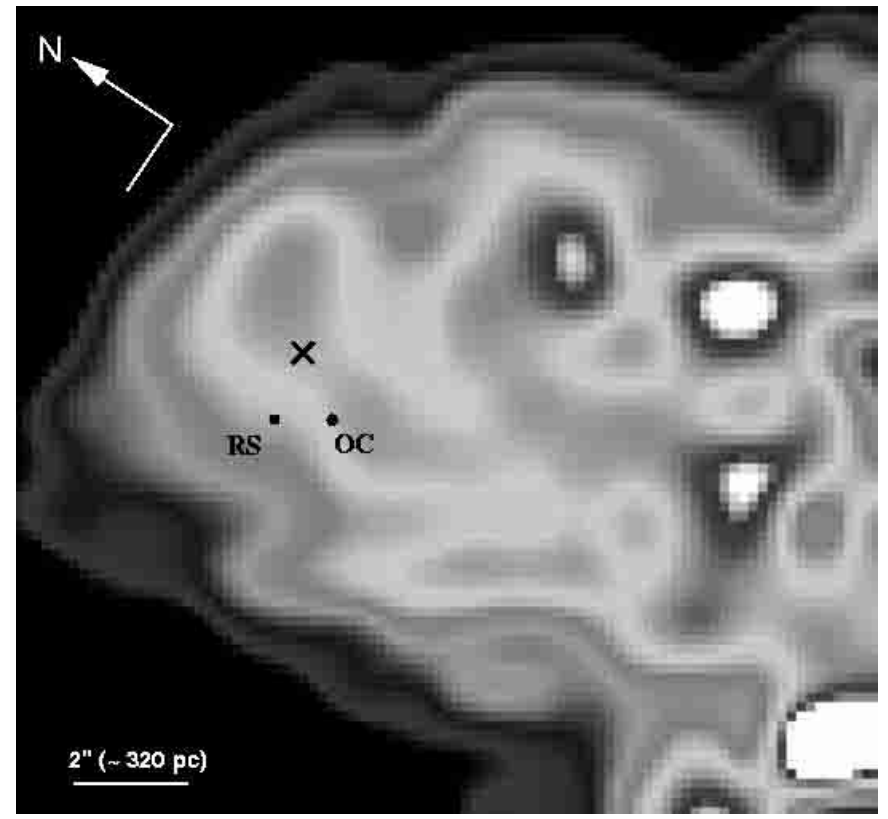

Fig. 4. The ionization cone obtained by the reconstructed map of the $[\mathrm{O} \mathrm{III}] / \mathrm{H} \beta$ emission line ratios. The $\times$ symbol indicates the position of the assumed nucleus (as in Fig. 1), OC is the maximum intensity of the optical continuum at $5500 \AA$, and RS is the approximate location of the radio source with flat spectrum.

producing an overcorrection of the metallic absorptions. This can happen since stellar populations cannot be omogeneus everywhere in the field of view. The resulting variations of line intensities have been estimated weak $(<10 \%$ for $\mathrm{H} \beta)$ in spectra with very bright emission lines, significant $(\sim 10-30 \%)$ in most of the spectra and very important in few spectra (up to $60 \%)$.

The emission line fluxes, measured after the starlight contamination removal, were corrected for internal reddening by means of the theoretical Balmer decrement value $\mathrm{H} \alpha / \mathrm{H} \beta=2.86$ for Case B recombination at electronic temperature $T_{\mathrm{e}}=10^{4} \mathrm{~K}$ and assuming the reddening law by Miller \& Mathews (1972).

Several images of the galaxy within the field of view were reconstructed at different wavelengths, as for instance the map of the visible continuum at $5500 \AA$. The position of its maximum intensity is located to the $\mathrm{N}-\mathrm{W}$ of the observed peak of the emission line maps, which we have decided to assume as position of the photoionization source (hereafter nucleus). We do not confirm the large offset between the location of the radio source with flat spectrum and the visible continuum peak indicated in Hummel \& Saikia (1991) and references therein: the radio source, the visible continuum and the emission lines have their maxima intensities close to each other (separation $\sim 1^{\prime \prime}$ ). We have discarded the idea to adopt the position of the radio source, which should indicate the real location of the AGN, as nucleus in our field of view since the spatial resolution of our data is only $1^{\prime \prime} / \mathrm{px}$ and an accurate astrometric calibration of the integral field images is very hard to obtain.

The map of the [O III] $\lambda 5007 / \mathrm{H} \beta$ emission line ratios revealed part of the S-W ionization cone (Fig. 4). The cone has the axis at $\mathrm{PA} \sim 200^{\circ}$ and an aperture angle of $\sim 70^{\circ}$, less

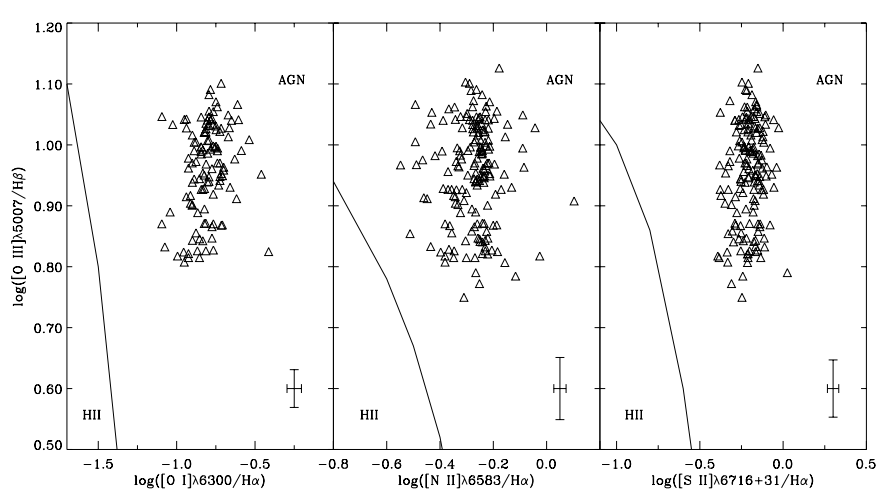

Fig. 5. Diagnostic diagrams of the emission line regions inside the ionization cone. The typical uncertainties of the ratios are indicated by the error bars in the bottom-right corner of each plot.

than the value measured by Pogge (1988), probably due to our smaller field of view.

The gaseous regions within the ionization cone have been investigated. Their intrinsic FWHM, estimated from the Gaussian analysis of the emission lines after having removed the instrumental component $(\sim 10 \AA)$, range in the interval $\sim 200-700 \mathrm{~km} \mathrm{~s}^{-1}$, with a median value of $\sim 480 \mathrm{~km} \mathrm{~s}^{-1}$, velocities usually observed in the Narrow Line Regions of Seyfert galaxies. The diagnostic emission line ratios [N II] $\lambda 6583 / \mathrm{H} \alpha$, [O I] $\lambda 6300 / \mathrm{H} \alpha$, [S II] $\lambda \lambda 6716+6731 / \mathrm{H} \alpha$ and [O III] $\lambda 5007 / \mathrm{H} \beta$ (Veilleux \& Osterbrock 1987), show the typical properties of gas ionized by a non-thermal source (Fig. 5).

Moreover the spatial distribution of the ionization degree within the cone, evaluated by means of the $[\mathrm{O}$ III $] \lambda 5007 / \mathrm{H} \beta$ ratio as a function of the distance from the nucleus, indicates that the gas is highly ionized even at large distances from the AGN. In order to understand if the AGN could account for such observed ionization, the $\mathrm{H} \alpha$ luminosity of each spectrum was calculated from the reddening corrected $\mathrm{H} \alpha$ fluxes. Then the number of ionizing photons needed to produce such luminosities, $Q_{\text {ion }}=7.3 \times 10^{11} \mathrm{~L}(\mathrm{H} \alpha)$ photons s${ }^{-1}$ (Kennicutt 1998), was evaluated and later compared with $Q_{\text {nuc }}^{\star}=Q_{\text {nuc }} \Omega / 4 \pi$, where $Q_{\text {nuc }}$ is the number of photons coming from the nucleus, diluted by the covering factor $\Omega / 4 \pi$, and $\Omega$ is the solid angle of a region seen from the nucleus.

By assuming that the nuclear source is an isotropic emitter of radiation occulted along our line of sight, we attempted to estimate $Q_{\text {nuc }}$ by taking the average of the calculated number of photons ionizing the regions surrounding the nucleus, after having removed the effect of the covering factor ( $Q_{\text {ion }} 4 \pi / \Omega$ ). A value of $Q_{\text {nuc }} \sim 4.87 \times 10^{52}$ photons s ${ }^{-1}$ was obtained, in good agreement with the $4.98 \times 10^{52}$ photons $\mathrm{s}^{-1}$ computed by Colina (1992) using the IUE spectrum of the galaxy and the flux at $912 \AA$.

The resulting $Q_{\text {ion }} / Q_{\text {nuc }}^{\star}$ ratio concentrates around a value of 2.5. This indicates that the active nucleus is the dominant ionizing source for the regions within our field of view, in agreement with what observed in the VO diagrams. In spite of that, the ionization parameter $U=Q_{\text {nuc }} / 4 \pi \mathrm{cr}_{0}^{2} N_{\mathrm{H}}$, calculated within the cone as a function of the distance $r_{0}$ from the nucleus, ranges between $10^{-5.0}$ and $10^{-2.8}$, a result clearly in 
contrast with the already pointed out high ionization level of almost all the regions. A total hydrogen density $N_{\mathrm{H}}=3 \times$ $10^{2} \mathrm{~cm}^{-3}$ is assumed, on the basis of the the electron density values estimated over the whole field, using the [S II] $\lambda \lambda 6717 / 6731$ ratio and assuming $T=10^{4} \mathrm{~K}$ (except where the sulphur lines were not visible or extremely blended). The problem of the missing data has been solved taking the average value of independent linear interpolations of the [S II] ratios along the two orthogonal directions of the spectral array. The resulting $N_{\mathrm{e}}$ distribution presented values $<10^{3} \mathrm{~cm}^{-3}$, with a median of $\sim 300 \mathrm{~cm}^{-3}$. Of course due to the uncertainties of the [S II] emission line strengths, the estimation of the electron density is not very accurate and just a simple decrease by a factor of 10 can account for the ionization parameter in the regions far from the nucleus.

\subsection{The models}

The contribution of the shocks to the line intensities is tested by comparing calculated with observed spectra. The code SUMA (Contini \& Viegas 2001, and references therein), which accounts consistently for the photoionization flux from an external source and for the shocks is adopted.

The general model assumes matter bound gaseous and dusty clouds, which move outwards from the source and emit the line and continuum spectra. A shock front forms on the outer edge of the clouds, while the inner edge is reached by the radiation flux from the source.

The input parameters are: the radiation flux intensity, $F_{\mathrm{H}}$ (in photons $\mathrm{cm}^{-2} \mathrm{~s}^{-1} \mathrm{eV}^{-1}$ at $\left.1 \mathrm{Ryd}\right)$, the spectral index, $\alpha(=1.5$ for all models), the shock velocity, $V_{\mathrm{s}}$, the preshock density, $n_{0}$, the preshock magnetic field, $B_{0}\left(=10^{-4}\right.$ Gauss $)$, the dust-togas ratio, $d / g$, and the geometrical thickness, $D$, of the emitting clouds. Cosmic relative abundances (Allen 1973) are adopted.

Composite models lead to a complex distribution of the temperature and the density throughout the clouds. In radiation dominated (RD) models the effect of the flux from the active center (AC) prevails on the shock, while in shock dominated (SD) models the flux is absent $\left(F_{\mathrm{H}}=0\right)$. When shocks and photoionizing fluxes act on the opposite sides of the cloud, two regions of relative high temperature appear near the edges (see Sect. 2.4). Higher temperatures correspond to higher shock velocities $\left(T \propto V_{\mathrm{s}}^{2}\right)$, while the radiation flux cannot heat the gas to $>2-3 \times 10^{4} \mathrm{~K}$. This temperature leads to high $\mathrm{O}^{+2} / \mathrm{O}$ oxygen fractional abundance, so the [OIII] line strength depends on $F_{\mathrm{H}}$.

The cooling rate ( $\propto n^{2}$, where $n$ is the density of the gas) increases with compression downstream of the shock leading to cool gas in the internal region of the cloud. The larger the cool region the strongest the low ionization level lines. The relative sizes of the hot and cool gas regions depend, therefore, on the shock velocity, on the intensity of the flux from the $\mathrm{AC}$, on the preshock density, and on the geometrical thickness of the clouds.

A large grid of models is calculated in order to select the most suitable parameters to the observational evidences for each region in the galaxy. Recall that a first choice is

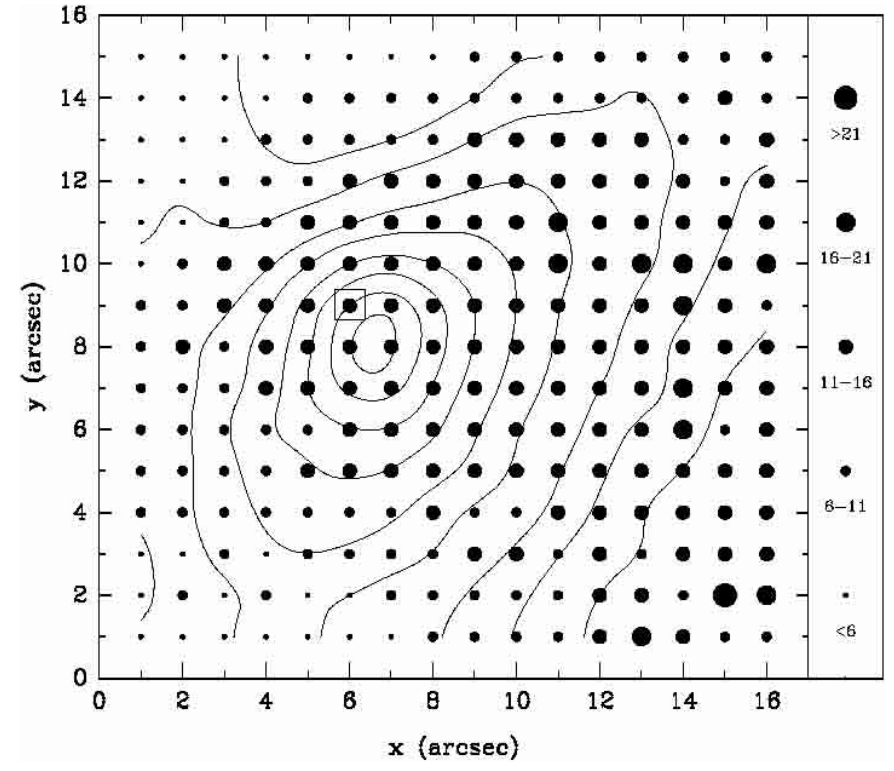

Fig. 6. Contour map of the visible continuum at $5500 \AA$. The isointensities correspond to the values: $1.2,1.5,1.8,2.2,2.5,2.8,3.0,3.15$, $3.25\left(\times 1 . e-15 \mathrm{erg} \mathrm{cm}^{-2} \mathrm{~s}^{-1}\right)$. Filled circles correspond to the values of the observed [O III] $\lambda \lambda 4959+5007 / \mathrm{H} \beta$ ratios. The small "squared window" located at $(6,9)$ indicates the assumed position of the ionization source. The map is orientated like in Fig. 4.

roughly obtained considering that the [O III] $\lambda 5007$ line flux depends on the intensity of the nuclear radiation flux, the [S II] $\lambda \lambda 6717 / 6731$ line ratio depends on the density, as seen before, and the intensity ratios of high to low ionization lines depend on the geometrical thickness of the clouds and on the shock velocity (see Contini \& Viegas 2001). The input parameters are then changed consistently in order to obtain the best fit of all the observed data.

\section{4. $[\mathrm{OIII}] / \mathrm{H} \beta$ and $[\mathrm{OI}] / \mathrm{H} \beta$}

The observations cover the SW cone providing line ratios in many different regions. We focus on [O III] $\lambda \lambda 4959+5007 / \mathrm{H} \beta$ and [O I] $\lambda \lambda 6300+6363 / \mathrm{H} \beta$ line ratios, because [O III] lines are generally strong in Seyfert 2 galaxies and are emitted by gas ionized by the radiation flux from the active nucleus, while [O I] lines are emitted from gas at lower temperatures and are generally strong in the presence of shocks.

Moreover, Veilleux et al. (1999a) claim that dynamical processes such as entrainment by AGN-powered radio jets generally have a stronger effect on the kinematics of the highly ionized [O III] emitting gas than on those of the low-ionization $\mathrm{H} \alpha$ emitting material.

Therefore, by modeling the observed spectra it is possible to determine the distribution of the radiation flux intensity and of other parameters, e.g. the shock velocity of the gas, the density, and the geometrical thickness of the clouds in the observed region. The reliability of the models is checked comparing the observed spectral energy distribution (SED) of the continuum with model results (see Sect. 4).

The observed [O III] $/ \mathrm{H} \beta$ line ratios overlaid to the continuum of the galaxy at $5500 \AA$ are shown in Fig. 6 in a synthetic representation. The $[\mathrm{O} \mathrm{III]}] / \mathrm{H} \beta$ distribution is not smooth. 


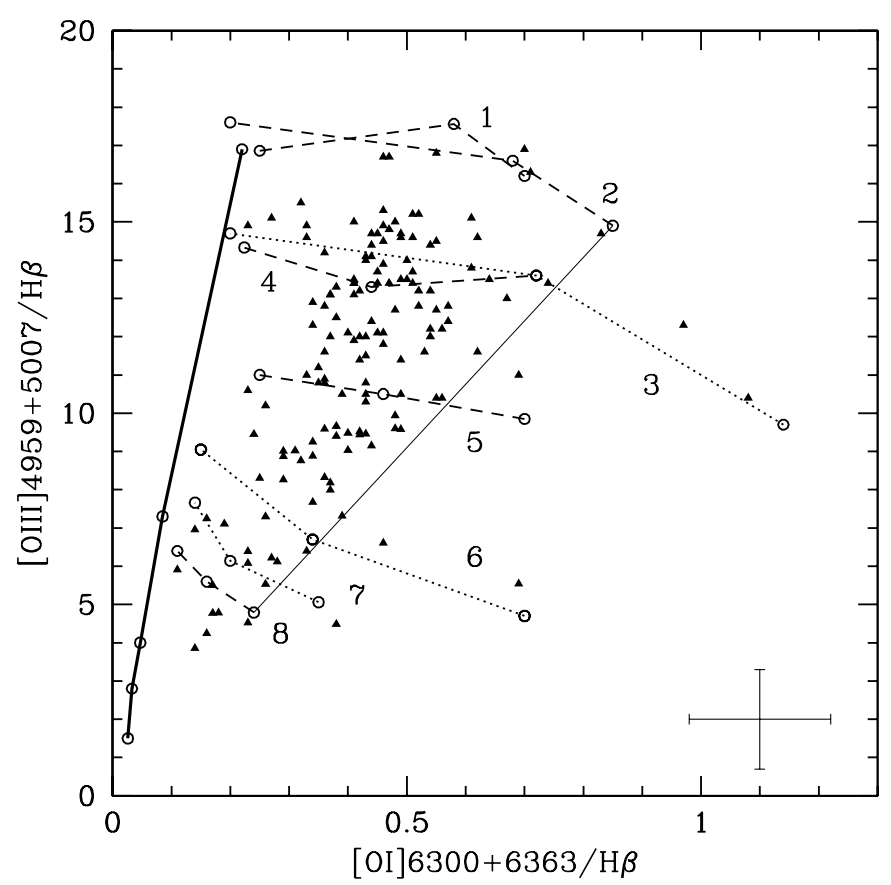

Fig. 7. The comparison of model calculation with observations (filled triangles) for $[\mathrm{O} \mathrm{III}] \lambda \lambda 4959+5007 / \mathrm{H} \beta$ vs $[\mathrm{O} \mathrm{I}] \lambda \lambda 6300+6363 / \mathrm{H} \beta$. See text for a detailed description.

Particularly, high $[\mathrm{O} \mathrm{III}] / \mathrm{H} \beta$ ratios appear in some regions far from the active center. This suggest that the clouds in those regions have abnormal intrinsic conditions.

In Fig. $7[\mathrm{O} \mathrm{III}] / \mathrm{H} \beta$ versus $[\mathrm{O} \mathrm{I}] / \mathrm{H} \beta$ from the observations (black triangles) is compared with model calculations. All the models correspond to $V_{\mathrm{s}}=100 \mathrm{~km} \mathrm{~s}^{-1}$. Higher velocities are less fitting, and velocities lower by a factor $<2$ lead to similar results. The thick solid line refers to shock dominated models with line ratios increasing with decreasing $D$ and $n_{0}$. The bulk of the data, however, is scattered throughout a region of the diagram better fitted by radiation dominated models. The thin solid line determines roughly the right edge of this region. The numbers in Fig. 7 refer to Table 1, where the models are described. The dashed lines connect models calculated with different $D$ (increasing from left to right) but all corresponding to the same flux intensity $F_{\mathrm{H}}$, while the dotted lines connect models calculated with different $F_{\mathrm{H}}$ (increasing from left to right) and corresponding to the same $D$. Figure 7 shows that the trend of both types of models is different from the observed one, i.e. $[\mathrm{O} \mathrm{III}] / \mathrm{H} \beta$ increasing with $[\mathrm{O} \mathrm{I}] / \mathrm{H} \beta$.

The increasing trend of the line ratios can be explained either by a slightly larger $D$ and stronger $F_{\mathrm{H}}$ (models 1 in Table 1$)$ or by very large clouds ( $D \geq 1 \mathrm{pc}$, models 2 in Table 1$)$, corresponding to a lower density, and a flux intensity not exceeding the characteristic values calculated for most of the observed regions inside the cone. The other observed lines at each position are too few and too uncertain to constrain the models, therefore, we suggest that the clouds reached by a strong nuclear flux (model 1) are located in the central region of the cone, while clouds showing the same high [OIII]/H $\beta$ ratios, but characterized by a low density and a large geometrical thickness (model 2), are located in some external regions of the cone.
Table 1. The models in Fig. 7.

\begin{tabular}{llll}
\hline \hline & $n_{0}\left(\mathrm{~cm}^{-3}\right)$ & $D\left(10^{17} \mathrm{~cm}\right)$ & $F_{\mathrm{H}}{ }^{1}$ \\
\hline 1 & 50. & $9,10,12$ & 1.5 \\
2 & 30. & $30,40,50$ & 0.9 \\
3 & 50. & 7. & $0.7,1.0,1.2$ \\
4 & 50. & $5,6,7$ & 1. \\
5 & 50. & $3.5,4,5$ & 0.7 \\
6 & 100. & 0.6 & $0.8,1.0,1.2$ \\
7 & 100. & 0.4 & $0.6,0.8,0.9$ \\
8 & 100. & $0.2,0.25,0.3$ & 0.6 \\
\hline
\end{tabular}

${ }^{1}$ In units of $10^{9}$ photons $\mathrm{cm}^{-2} \mathrm{~s}^{-1} \mathrm{eV}^{-1}$ at $1 \mathrm{Ryd}$.

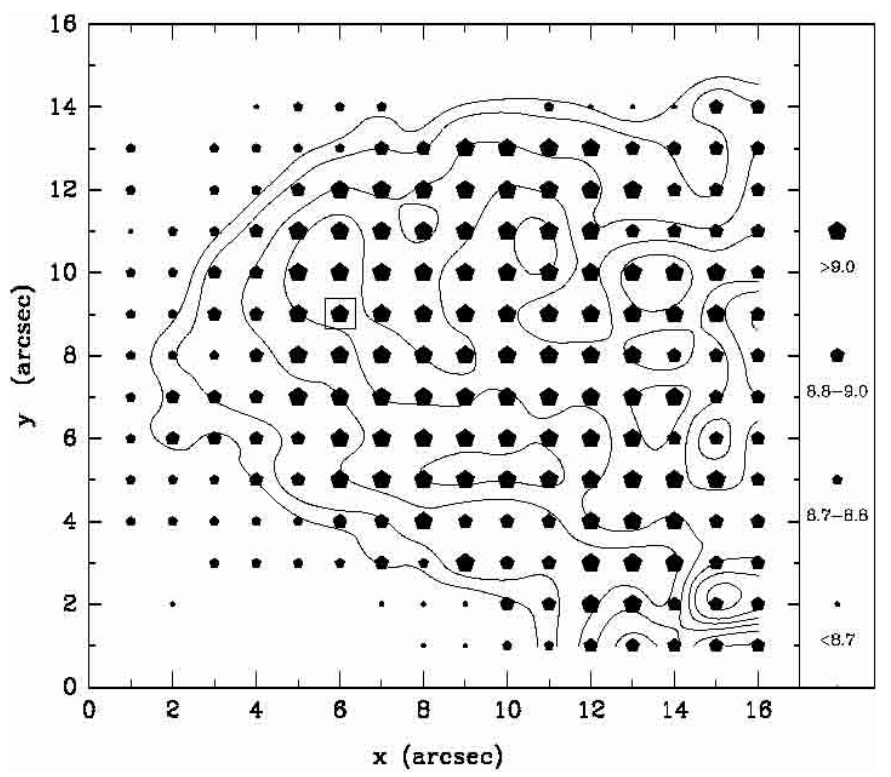

Fig. 8. The grid of the $F_{\mathrm{H}}$ values (filled pentagons) overlaid to the contour map of the ionization cone. The isointensities correspond to the $[\mathrm{O}$ III] $] \lambda \lambda 4959+5007 / \mathrm{H} \beta$ ratios: $9.5,10.5,12.5,14,15.5,17,19$. The map is oriented as in Fig. 4. The square is like in Fig. 6.

Extended clouds of rarefied gas appear in the terminal region of jets and winds.

The distribution of $F_{\mathrm{H}}$ in the different regions of the cone, as it results from modeling is shown in Fig. 8. The range of the corresponding ionization parameters $U$ is $2 \times 10^{-5}-5 \times 10^{-4}$.

To better understand the results obtained above, the distributions of the electron temperature (dotted line) and density (solid line) throughout a cloud are given for different models in Figs. 9 and 10. Each figure is divided by a solid vertical line in two halves. The horizontal axis scale is logarithmic and symmetric to have a comparable view of the two sides of the cloud. The shock front is on the left and the radiation flux from the source reaches the right edge.

The geometrical thickness $D$ is a crucial parameter to lowlevel and, particularly, to neutral lines, because they are emitted from rather cold gas. Therefore, in the bottom pannels the distribution of the fractional abundance of $\mathrm{O}^{+0} / \mathrm{O}$ (dotted line), $\mathrm{O}^{+1} / \mathrm{O}$ (short-dash line), $\mathrm{O}^{+2} / \mathrm{O}$ (long-dash line), and $\mathrm{H}^{+1} / \mathrm{H}$ (solid line) have been plotted. Figure 9 corresponds to 


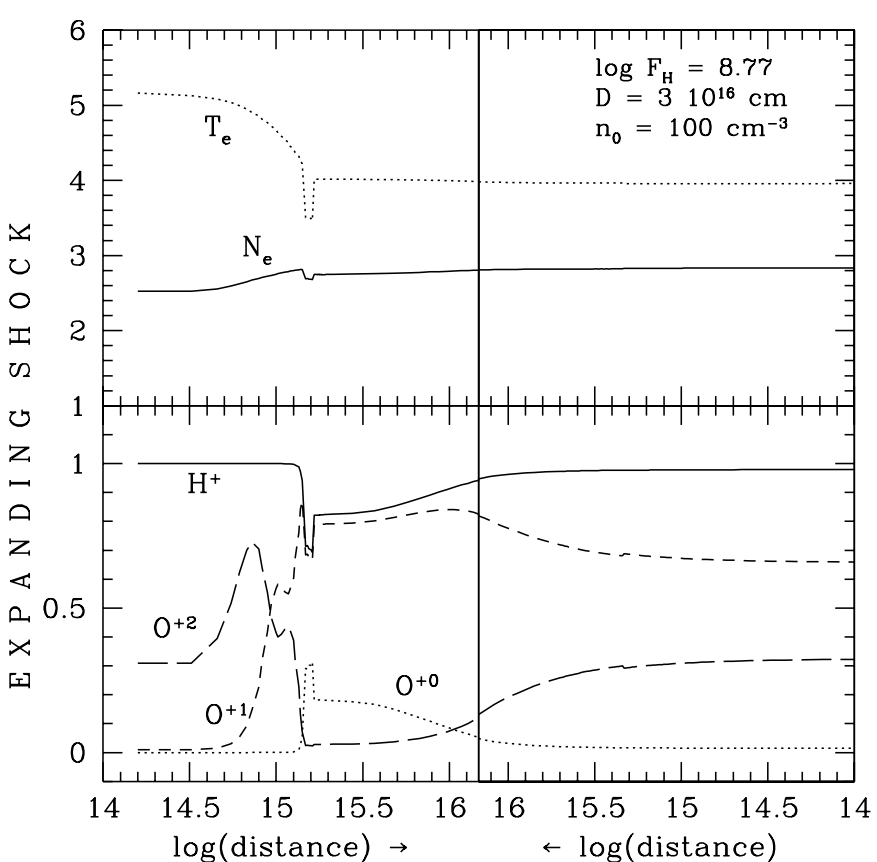

Fig. 9. The case of a small $D$ and relatively low $F_{\mathrm{H}}$. The distribution of the temperatures and densities (upper panels) and of the most significant ion fractional abundances (lower pannels) throughout the clouds for different models (see text).

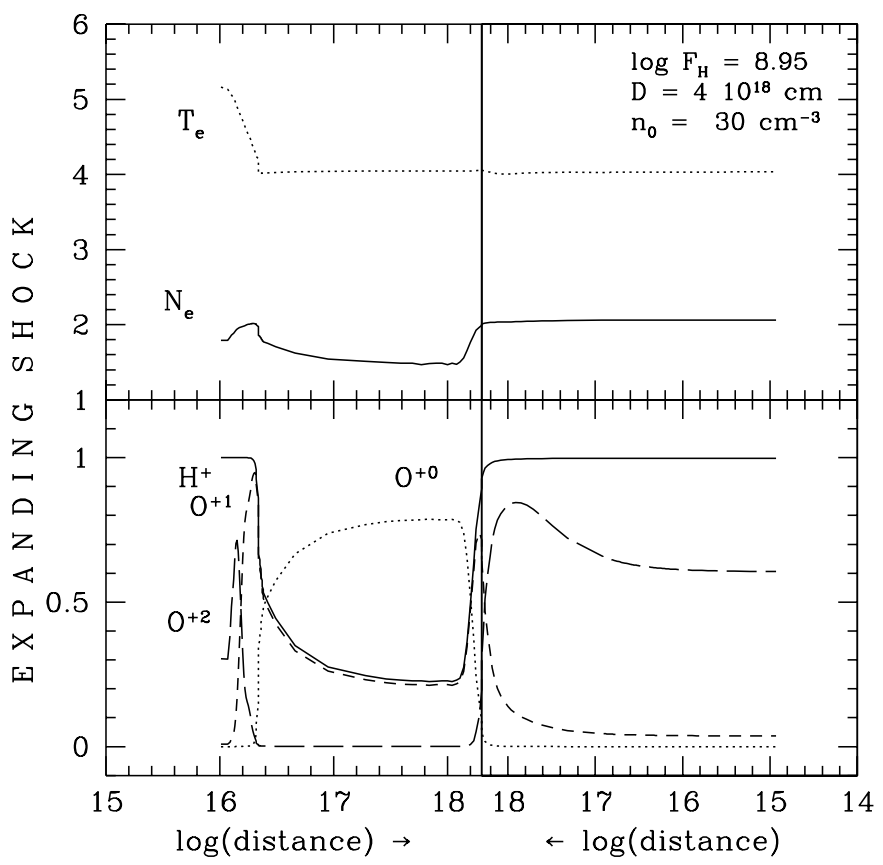

Fig. 10. The same as for Fig. 9 in the case of a large $D$ and low $n_{0}$.

$D=3 \times 10^{16} \mathrm{~cm}(0.01 \mathrm{pc})$ and Fig. 10 to $D=4 \times 10^{18} \mathrm{~cm}$ $(1.3 \mathrm{pc})$. It can be noticed that two zones of gas emit the [O III] lines, one from the side of the shock, where collisional ionization prevails, and the other from gas ionized by radiation. The ionization rate due to the photoionization flux from the nuclear source, dominates in a large region of the cloud, particularly in models calculated with $V_{\mathrm{s}}=100 \mathrm{~km} \mathrm{~s}^{-1}$, where the gas density and optical thickness are rather low. A large region of cool gas appears inside clouds corresponding to a large

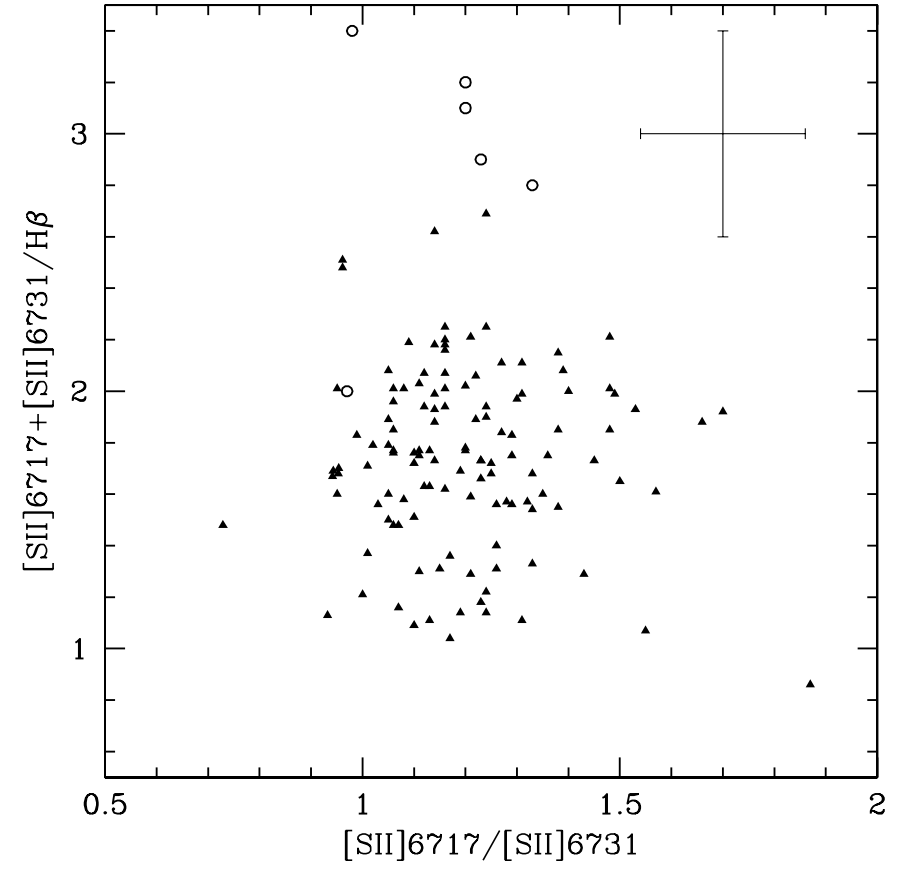

Fig. 11. The same as for Fig. 7 for $[\mathrm{S} \mathrm{II}] \lambda \lambda 6716+6731 / \mathrm{H} \beta$ vs. [S II] $\lambda \lambda 6716 / 6731$.

geometrical thickness and low density, leading to relatively strong [O I] lines.

\section{5. $[S I I] / H \beta$}

Figure 11 shows [S II] $\lambda \lambda 6717+6731 / \mathrm{H} \beta$ versus [S II] $\lambda \lambda 6717 / 6731$. The low ionization lines are strongly affected by shocks. For models accounting for the shock a large range of electron densities follows from compression in the downstream region. The lines, calculated integrating through the geometrical thickness of the clouds, account for the different conditions of the gas (see Sect. 2.4).

Some significant models, which are the same as those adopted to explain the $[\mathrm{O} \mathrm{III}] / \mathrm{H} \beta$ ratios in Fig. 7 , are indicated by open circles. They show a good agreement to the observed [S II] $\lambda \lambda 6717 / 6731$ line ratio between 1 and 1.3 in Fig. 11. The low density model gives [S II] $\lambda \lambda 6717 / 6731 \leq 1.4$. Notice, however, that the [S II] $\lambda \lambda 6717+6731 / \mathrm{H} \beta$ ratios are higher by a factor $<2$ than the data for most models, indicating that the $\mathrm{S} / \mathrm{H}$ relative abundance is lower than cosmic. This is a common characteristic to Seyfert 2 galaxies where sulphur is depleted from the gaseous phase. The models are calculated adopting $d / g$ (by number) $=10^{-14}-10^{-13}$, indicating that dust is indeed present in the NGC 4388 cone region.

Reminding that compression increases with $V_{\mathrm{s}}$, models calculated with higher densities and velocities $\left(V_{\mathrm{s}}=300 \mathrm{~km} \mathrm{~s}^{-1}\right)$ fit the data corresponding to low [S II] $\lambda \lambda 6717 / 6731(<1)$, although their observed errors are large. Finally, models with $V_{\mathrm{s}}=500 \mathrm{~km} \mathrm{~s}^{-1}$ hardly fit any data, suggesting that high velocity clouds are relatively few (see Sect. 4).

The results show that the bulk of the shock velocities is lower than the velocities corresponding to the FWHM of the emission lines (200-700 $\mathrm{km} \mathrm{s}^{-1}$ ), indicating that strong shocks 
do not form in the observed region of NGC 4388, but rather low shock velocities $V_{\mathrm{s}}=100 \mathrm{~km} \mathrm{~s}^{-1}$ and low densities $n_{0} \leq$ $100 \mathrm{~cm}^{-3}$ dominate.

\section{The North-East cone}

We now consider the $\mathrm{N}-\mathrm{E}$ cone and the very extended emission-line region (VEELR) discovered by YOS02 in order to have a larger view of the ionization conditions in NGC 4388. The nuclear region of the galaxy has been successfully investigated by Petitjean \& Durret (1993), but only a few emission lines were observed at $21^{\prime \prime}, 25^{\prime \prime}$, and $38^{\prime \prime}$ from the nucleus.

YOS02 observed the [O III] $\lambda 5007$ and $\mathrm{H} \alpha+[\mathrm{N} \mathrm{II}]$ $\lambda \lambda 6548,6583$ fluxes from different clouds in the VEELR, which extends northeastward of the galaxy, very far outside the galaxy disk. The ionization cone is traced by the N-E plume and a high ionization cloud located at $10^{\prime \prime}-15^{\prime \prime}$ northwest of the nucleus. They claim that there is a sudden change in the excitation state of the gas around the line at PA $65^{\circ}$, which coincides with the extrapolation of the northern edge line of the $\mathrm{S}-\mathrm{W}$ cone. Notice that these authors assume as distance of NGC 4388, the distance of the Virgo cluster $(16.7 \mathrm{Mpc})$, which is different from that one used by us for the S-W cone $(33.6 \mathrm{Mpc})$. Therefore, all the distances should be scaled by a factor of 2 .

In Figs. 12-14 we compare the results of models calculated with the code SUMA with the YOSO2 data. The errorbars refer to the typical uncertainty of $20 \%$ estimated by YOS02. In all the diagrams the fluxes of [O III] versus $\mathrm{H} \alpha+[\mathrm{N} \mathrm{II}]$ (in erg cm${ }^{-2} \mathrm{~s}^{-1}$ ) are presented. Recall that models are calculated at the emitting nebula, while data are observed at Earth. Therefore, we have multiplied the data by $d^{2} / r^{2}$, where $r$ is the distance of each cloud from the center of the galaxy and $d$ is the distance of NGC 4388 from Earth.

All models are calculated adopting a geometrical thickness, $D=3 \mathrm{pc}$, and $B_{0}=10^{-5}$ Gauss, which is similar to the magnetic field in the ISM $\left(B_{0}=10^{-6}-10^{-5}\right.$ Gauss).

Shock dominated models, calculated considering only heating and ionization of the clouds by the shock $\left(F_{\mathrm{H}}=0\right)$, are presented in Fig. 12. The models are labeled by the shock velocity in $\mathrm{km} \mathrm{s}^{-1}$. Large squares correspond to $n_{0}=10 \mathrm{~cm}^{-3}$, small squares to $n_{0}=2 \mathrm{~cm}^{-3}$. Relatively low preshock densities lead to the best agreement with the data, however, the SD models give a poor fit to the observed trend. This confirms YOS02 suggestion that gas excitation depends also on the flux from the active nucleus even at such large distances. We have used, therefore, RD models to fit the data. Recall that RD models are also composed because they account for the shock, even if the effect of the photoionizing flux prevails.

We present in Figs. 13 and 14 radiation dominated models calculated with different velocities and fluxes, adopting $n_{0}=10 \mathrm{~cm}^{-3}$ and $2 \mathrm{~cm}^{-3}$, respectively. Higher densities do not fit. In Fig. 13 black triangles indicate models calculated with $V_{\mathrm{s}}=50 \mathrm{~km} \mathrm{~s}^{-1}$, white triangles in the left side of the diagram corresponding to $V_{\mathrm{s}}=30 \mathrm{~km} \mathrm{~s}^{-1}$ and at the right to $V_{\mathrm{s}}=70-90 \mathrm{~km} \mathrm{~s}^{-1}$. The models are labeled by the values of $\log \left(F_{\mathrm{H}}\right)$. The maximum [N II] $+\mathrm{H} \alpha$ value is explained by $V_{\mathrm{s}}=150 \mathrm{~km} \mathrm{~s}^{-1}$. Dotted lines join the results of models

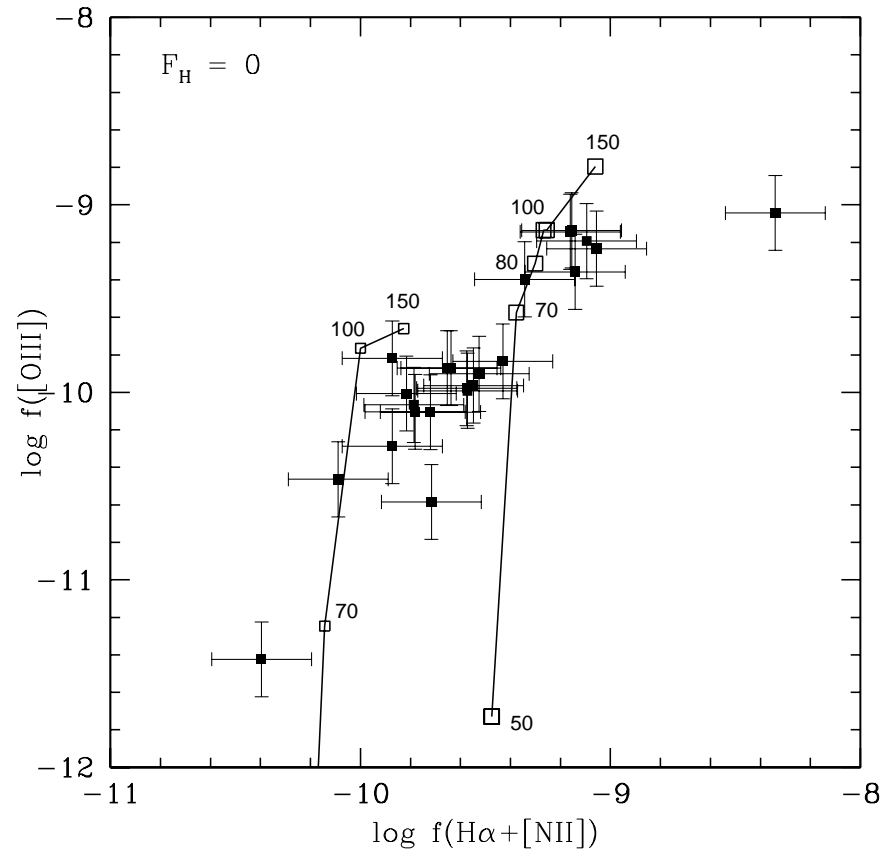

Fig. 12. The comparison of model results (corresponding to $F_{\mathrm{H}}=0$.) with the observations for [O III] $\lambda 5007\left(\mathrm{erg} \mathrm{cm}^{-2} \mathrm{~s}^{-1}\right)$ versus $[\mathrm{N} \mathrm{II}]+\mathrm{H} \alpha\left(\mathrm{erg} \mathrm{cm}^{-2} \mathrm{~s}^{-1}\right)$ in the VEELR. Symbols are given in the text.

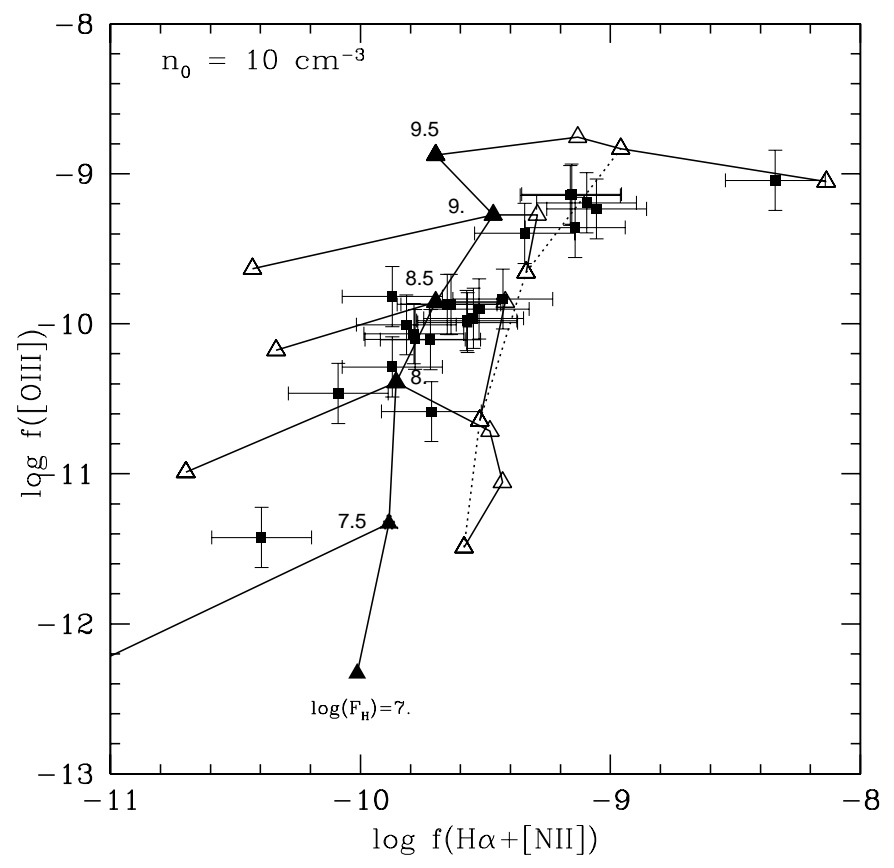

Fig. 13. The same as for Fig. 12 for composite models calculated with $n_{0}=10 \mathrm{~cm}^{-3}$.

calculated with $V_{\mathrm{s}}=90 \mathrm{~km} \mathrm{~s}^{-1}$. Notice that, roughly, all the data can be reached by the models represented by the grid.

In Fig. 14 the models are calculated with $V_{\mathrm{s}}=50 \mathrm{~km} \mathrm{~s}^{-1}$ (black triangles), $V_{\mathrm{s}}=70 \mathrm{~km} \mathrm{~s}^{-1}$ (small white triangles), and $V_{\mathrm{s}}=100 \mathrm{~km} \mathrm{~s}^{-1}$ (large white triangles). The adopted fluxes range between $\log F_{\mathrm{H}}=7$ and 9. It can be noticed that the calculated trends do not correspond to the observed one, however, some data are well reproduced by the models. In particular, the 


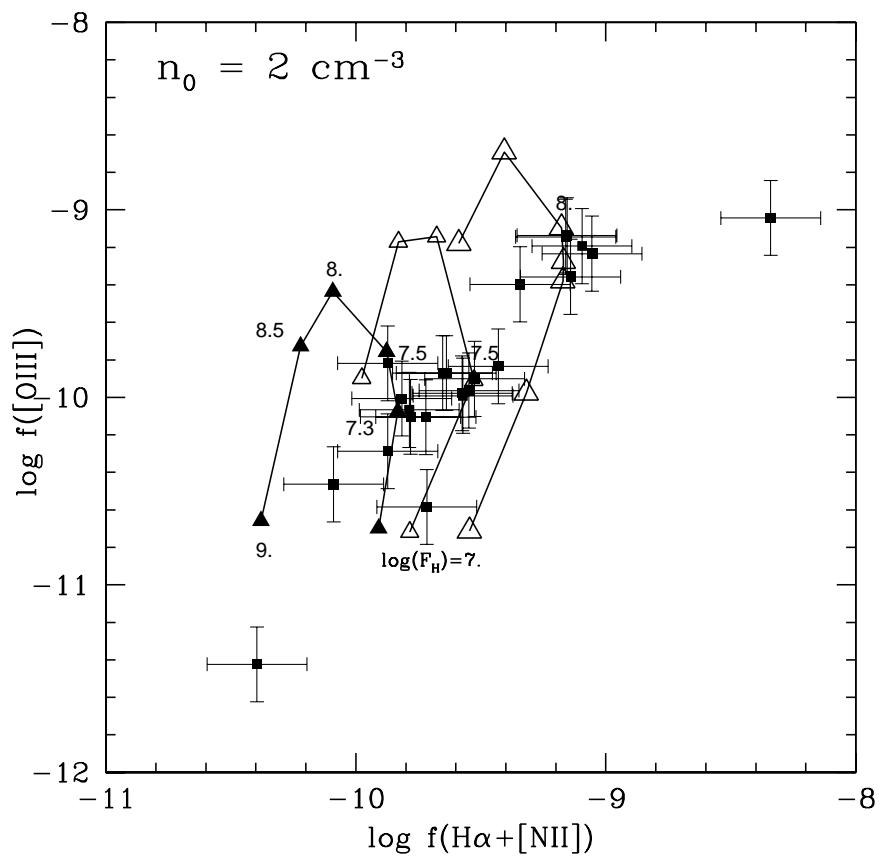

Fig. 14. The same as for Fig. 13 for composite models calculated with $n_{0}=2 \mathrm{~cm}^{-3}$.

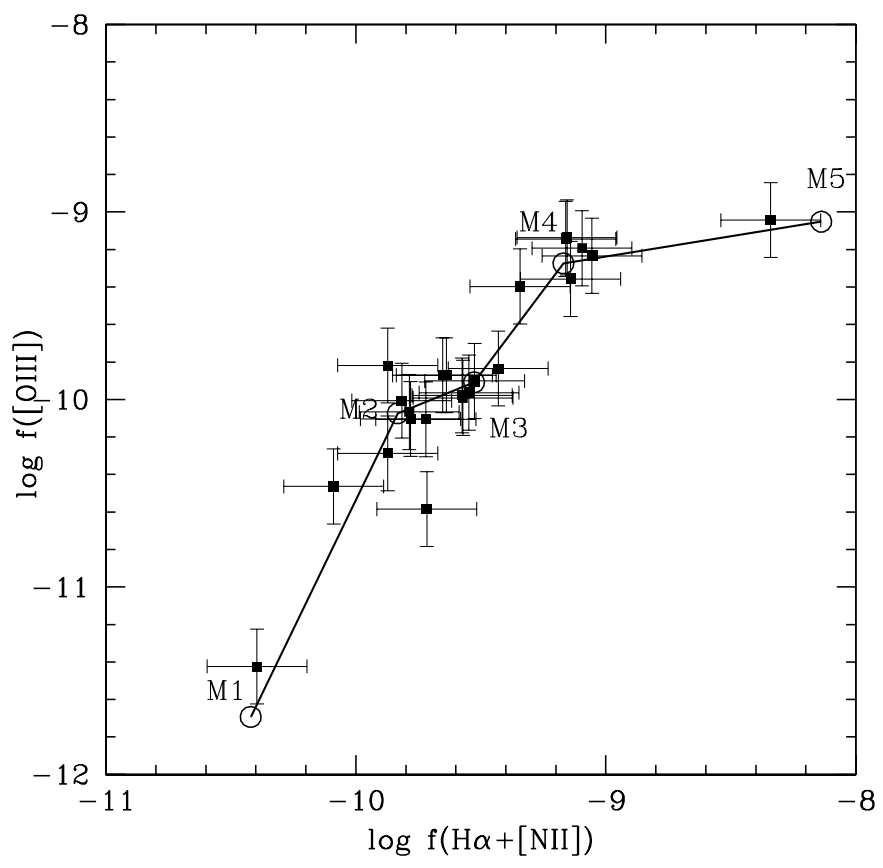

Fig. 15. The comparison of selected model results with the observations for [O III] $\lambda 5007\left(\mathrm{erg} \mathrm{cm}^{-2} \mathrm{~s}^{-1}\right)$ versus [N II] $+\mathrm{H} \alpha\left(\mathrm{erg} \mathrm{cm}^{-2} \mathrm{~s}^{-1}\right)$ in the VEELR (see text).

maximum and minimum observed [N II] $+\mathrm{H} \alpha$ are not explained by these models.

We have chosen the best fitting models from Figs. 13 and 14 and we present in Fig. 15 consistent modeling of the entire dataset. The selected models represented by open circles are described in Table 2.

The fluxes of [O III $]$ and $\mathrm{H} \alpha+[\mathrm{N} \mathrm{II}]$ are given in Fig. 16 as function of distance from the center. The data refer to the fluxes observed at the clouds. Figure 16 shows that the line fluxes
Table 2. The best fitting models.

\begin{tabular}{llllll}
\hline \hline & $M 1$ & $M 2$ & $M 3$ & $M 4$ & $M 5$ \\
\hline$n_{0}\left(\mathrm{~cm}^{-3}\right)$ & 2. & 2. & 2. & 2. & 10. \\
$V_{\mathrm{s}}\left(\mathrm{km} \mathrm{s}^{-1}\right)$ & 35. & 50. & 70. & 100. & 150. \\
$\log \left(F_{\mathrm{H}}\right)$ & 6.5 & 7.3 & 7.5 & 7.9 & 9.5 \\
\hline
\end{tabular}

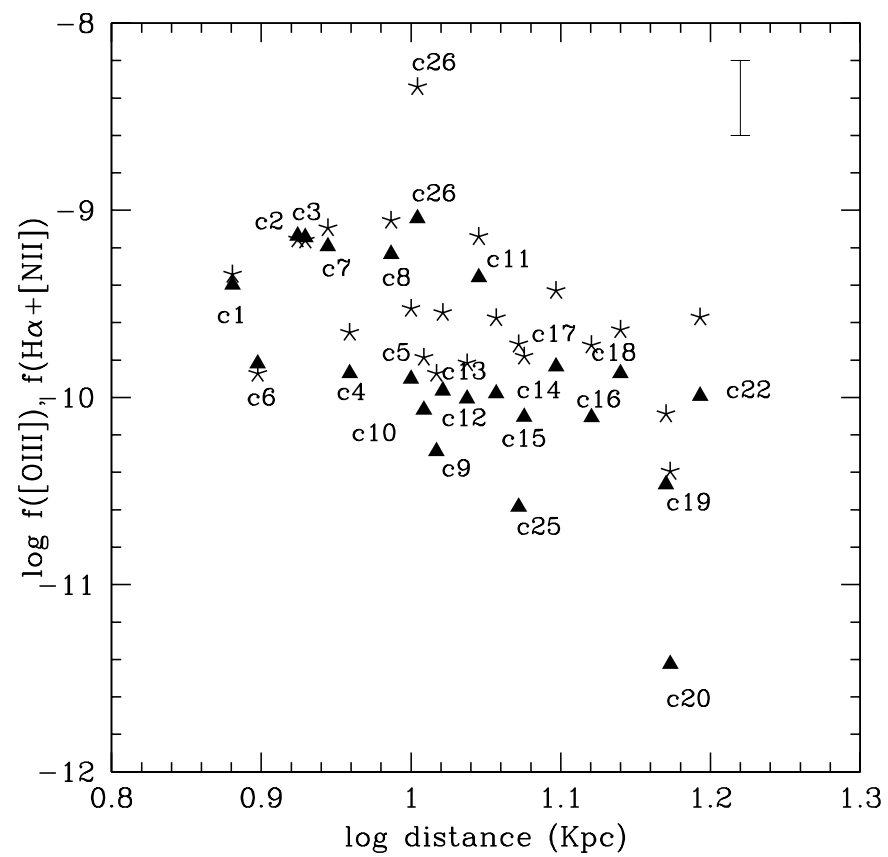

Fig. 16. The fluxes of [O III] (filled triangles) and [N II] $+\mathrm{H} \alpha$ (asterisks) observed at the clouds are plotted as function of the cloud distance from the center.

decrease with distance, however, different conditions coexist in the VEELR leading to the large scattering.

The emission line flux from the cloud, identified by YOSO2 (see their Fig. 3) as C20, located at the extreme northern edge of the cone, is explained by a low flux $\left(\log F_{\mathrm{H}}=6.5\right)$, $n_{0}=2 \mathrm{~cm}^{-3}$, and a low shock velocity $\left(35 \mathrm{~km} \mathrm{~s}^{-1}\right)$. The largest $\mathrm{H} \alpha+[\mathrm{N} \mathrm{II}]$ flux is emitted by the cloud identified as C26, located in the nebula at the southern edge of the eastern cloud. It corresponds to relatively high $F_{\mathrm{H}}\left(\log F_{\mathrm{H}}=9.5\right)$ and high shock velocity $\left(\leq 150 \mathrm{~km} \mathrm{~s}^{-1}\right)$. Also cloud $\mathrm{C} 25$ is slightly dislocated from the correlating curve in Fig. 15. Therefore, it shows conditions different from those of the other clouds. Cloud $\mathrm{C} 1$ shows the lowest distance from center and is modeled by $\log F_{\mathrm{H}}=9$, $V_{\mathrm{s}}=70 \mathrm{~km} \mathrm{~s}^{-1}$, and $n_{0}=10 \mathrm{~cm}^{-3}$ (Fig. 11). However, C1 also corresponds to a shock dominated model (Fig. 12) with $V_{\mathrm{s}}=80 \mathrm{~km} \mathrm{~s}^{-1}$ and $n_{0}=10 \mathrm{~cm}^{-3}$. We have not enough data to constrain the model and we conclude that many different conditions coexist in the clouds corresponding to the observations; in fact, the flux from the active nucleus, screened by the intervening matter, cannot reach all of the filaments within the observed clouds. The geometrical thickness adopted in the models is $D=3 \mathrm{pc}$, while the observed clouds show dimensions of the order of $100 \mathrm{pc}$. Shock dominated models are radiation bound, so the results should not change even adopting lower geometrical thickness. 
To fit the data in Figs. 12-14, we multiply the models by factors of $10^{-5}, 10^{-8}$ and $10^{-6.5}$, respectively. They account for the filling factor. Cloud $\mathrm{C} 20$ has a surface area of $S=60 \times 190 \mathrm{pc}^{2}$ and the distance from center is $r=14.9 \mathrm{kpc}$ (Yoshida et al. 2002, Table 2). From $S /\left(4 \pi r^{2}\right) \times f f=10^{-6.5}$ the filling factor results $f f=0.08$. For cloud $\mathrm{C} 26$, which corresponds to gas with a higher density, we obtain $f f=1.3 \times 10^{-4}$. Regarding cloud $\mathrm{C} 1, f f=1.4 \times 10^{-4}$ results for the radiation dominated filaments and $f f=0.14$ for the shock dominated filaments.

YOS02 claim that the inner region $(r<12 \mathrm{kpc})$ of the VEELR may be excited by nuclear ionizing radiation, while the excitation mechanism of the outer region $(r>12 \mathrm{kpc})$ is unclear. Our analysis is not complete because the [O III] line fluxes were not observed for $r>15.6 \mathrm{kpc}$. It is found, however, that clouds C19-C22 correspond to lower fluxes and lower velocities, because the nuclear flux from the AC is diluted by distance. The ionization parameter $U$ that results from modeling the VEELR ranges between $10^{-5}$ and $1.5 \times 10^{-3}$. The shock velocities $\left(50-150 \mathrm{~km} \mathrm{~s}^{-1}\right)$ are in agreement with the radial velocities in the N-E plume, i.e. 100$200 \mathrm{~km} \mathrm{~s}^{-1}$ (Yoshida et al. 2002).

\section{The SED of the continuum}

The consistency of the models is checked in Fig. 17 where the SED of the bremsstrahlung radiation and re-radiation by dust from different clouds within NGC 4388 is compared with the data. Therefore, in Fig. 17 two lines correspond to each model, one indicating the bremsstrahlung from the gas and the other emission from dust in the infrared. The observational data (from the $\mathrm{NED}^{2}$ ) are taken from Boroson et al. (1983), Scoville et al. (1983), de Vaucouleurs \& Longo (1988), Soifer et al. (1989), Moshir et al. (1990), Becker et al. (1991), de Vaucouleurs et al. (1991), Gregory \& Condon (1991), Kinney et al. (1993), Fabbiano et al. (1992), Zwicky et al. (1961), Douglas et al. (1996), Dressel \& Condon (1978), Spinoglio et al. (1995) and Mould et al. (1980). The two data in the soft X-ray range come from Einstein (Fabbiano et al. 1992), integrated on $0.2-4.0 \mathrm{keV}$, and from ROSAT (Halderson et al. 2001), integrated on 0.1-2.4 keV.

Indeed, the data from the NED were taken with different precisions and in different epochs. Nevertheless, they are very significant because they correspond to the main contributions to the continuum in the different frequency ranges by (1) bremsstrahlung from the photoionized gas $\left(v \sim 5 \times 10^{13}\right.$ $5 \times 10^{15} \mathrm{~Hz}$ ), (2) bremsstrahlung from the hot gas in the downstream region of shocked clouds $\left(v>10^{16} \mathrm{~Hz}\right)$, (3) emission from the old star population $\left(v \sim 5 \times 10^{13}-10^{15} \mathrm{~Hz}\right)$, (4) dust reradiation in the infrared range $\left(v \sim 10^{12}-10^{14} \mathrm{~Hz}\right)$, and (5) radio emission $\left(v<10^{11} \mathrm{~Hz}\right)$.

Besides the line and continuum spectra, the SUMA code calculates reradiation from dust in a consistent way. Dust is heated by the nuclear radiation flux; moreover, gas and dust mutually heat each other by collisions in shock turbulent regimes (Viegas \& Contini 1994). Therefore, the frequency

\footnotetext{
${ }^{2}$ NASA/IPAC Extragalactic Database.
}

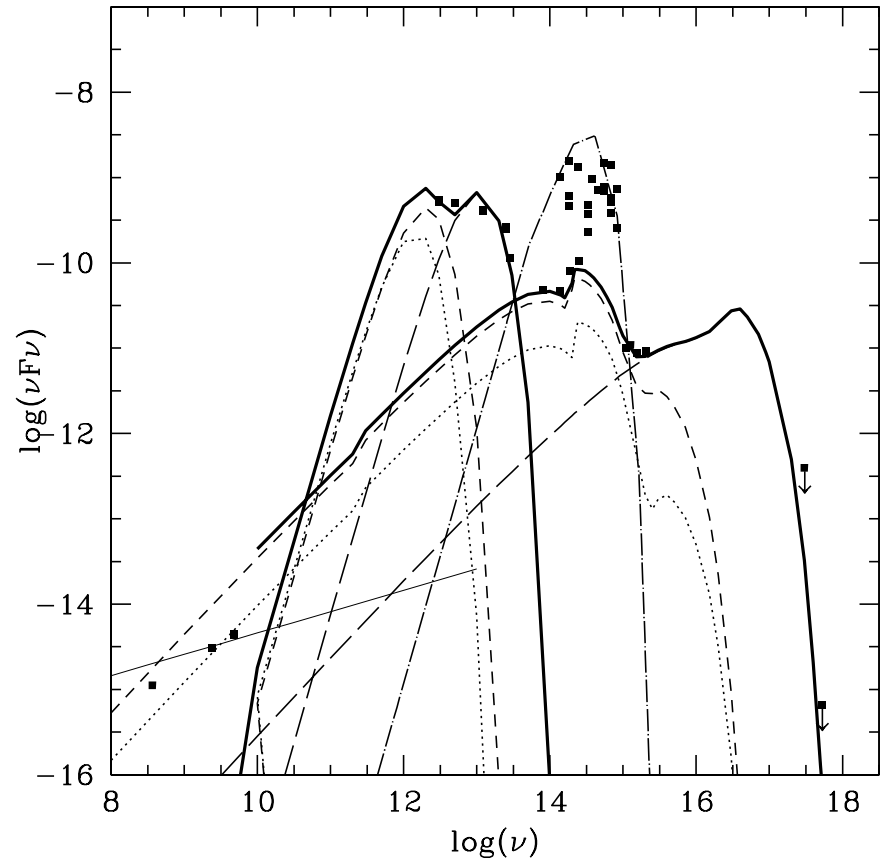

Fig. 17. The SED of the continuum. The data are represented by filled squares. $V_{\mathrm{s}}=100 \mathrm{~km} \mathrm{~s}^{-1}, n_{0}=100 \mathrm{~cm}^{-3}, \log F_{\mathrm{H}}=8.77, D=0.01 \mathrm{pc}$, $d / g=1.5 \times 10^{-14}$ (short-dash lines), $V_{\mathrm{s}}=100 \mathrm{~km} \mathrm{~s}^{-1}, n_{0}=30 \mathrm{~cm}^{-3}$, $D=1.3 \mathrm{pc}, \log F_{\mathrm{H}}=8.95 \mathrm{~d} / \mathrm{g}=10^{-14}$ (dotted lines), and $V_{\mathrm{s}}=$ $500 \mathrm{~km} \mathrm{~s}^{-1}, n_{0}=300 \mathrm{~cm}^{-3}, D=3.3 \mathrm{pc}, d / g=1.5 \times 10^{-13}$ (longdash lines). The summed continuum SED (thick solid lines); radio synchrotron continuum (thin solid line).

corresponding to the dust reradiation peak depends on the shock velocity. At high $V_{\mathrm{s}}$ the grains will reach high temperatures in the post shock region and dust reradiation will peak in the mid-IR, while at low velocities the peak appears in the far-IR. The peak intensity depends on the dust-to-gas ratio.

Sputtering of the grains, which depends on the shock velocity, is calculated throughout the clouds. Small grains are rapidly sputtered, therefore we adopt silicate grains with an initial radius of $0.2 \mu \mathrm{m}$.

Emission in the radio range corresponds to bremsstrahlung from cool gas as well as to synchrotron radiation due to Fermi mechanism at the shock front.

The continuum SED corresponding to single clouds are shown in Fig. 17 in order to explain the role of the different models. Then, the contributions from all the clouds are summed up to fit the data consistently in all the frequency domains.

The models obtained for the $\mathrm{S}-\mathrm{W}$ cone are used to model the SED of NGC 4388, because the emission in the VEELR is very low and can hardly affect the continuum observed from the entire galaxy. The models presented in Fig. 17 are selected from those which better fit the bulk of the data shown in Fig. 7 . We will consider three significant models, regarding the shock velocity, the preshock density, the geometrical thickness of the cloud, and the dust-to-gas ratio. The short-dashed lines refer to a model calculated with $V_{\mathrm{s}}=100 \mathrm{~km} \mathrm{~s}^{-1}, n_{0}=100 \mathrm{~km} \mathrm{~s}^{-1}$, $D=0.17 \mathrm{pc}$ and $d / g=1.5 \times 10^{-14}$. Notice that $d / g=10^{-14}$ by number corresponds to $4.1 \times 10^{-4}$ by mass (Draine $\&$ Lee 1984) which is the dust-to-gas ratio in the Galaxy adopting a 
silicate density of $3 \mathrm{~g} \mathrm{~cm}^{-3}$. Dotted lines represent the SED of the model calculated with $D=10 \mathrm{pc}, n_{0}=30 \mathrm{~cm}^{-3}$, $\log F_{\mathrm{H}}=9.8$, and $d / g=10^{-14}$. Considering that the FWHM of the line profiles show velocities up to $\geq 500 \mathrm{~km} \mathrm{~s}^{-1}$, we have added in Fig. 17 also the continuum of a SD model corresponding to $V_{\mathrm{s}}=500 \mathrm{~km} \mathrm{~s}^{-1}, n_{0}=300 \mathrm{~cm}^{-3}$, and $d / g=1.5 \times 10^{-13}$, in order to fit the data at higher frequencies. This relatively high shock velocity leads to a temperature in the immediate postshock region of $\sim 3.7 \times 10^{6} \mathrm{~K}$. Consequently bremsstrahlung radiation peaks in the soft $\mathrm{X}$-ray domain.

Indeed, the analysis of the ROSAT HRI observations by Matt et al (1994) has shown that the 0.1-2.4 keV X-ray emission is dominated by an extended component $(\sim 4.5 \mathrm{pc})$. It is interpreted as thermal emission from a collisionally heated plasma and a collection of discrete sources. The corresponding temperature of $\sim 0.4 \mathrm{keV}\left(\sim 4.6 \times 10^{6} \mathrm{~K}\right)$ is in agreement with the temperature of $\sim 4 \times 10^{6} \mathrm{~K}$ calculated by $V_{\mathrm{s}}=500 \mathrm{~km} \mathrm{~s}^{-1}$. No correlation of the soft X-ray emission with the optical ionization cones has been found (Matt et al.). This confirms our previous results (Sects. 2 and 3 ) showing that rather low $\left(\sim 100 \mathrm{~km} \mathrm{~s}^{-1}\right)$ shock velocities dominate in the cones.

Figure 17 shows that the SED of single models calculated with $V_{\mathrm{s}}=100 \mathrm{~km} \mathrm{~s}^{-1}$ nicely fit the data corresponding to the lowest fluxes in the optical-UV range. The data between $10^{14}$ and $10^{15} \mathrm{~Hz}$ are, however, contaminated by radiation from old population stars and are explained by a black body model corresponding to $5 \times 10^{3} \mathrm{~K}$ (dash-dotted line). This is common to the continua of Seyfert 2 galaxies (Contini \& Viegas 2000).

Recall that dust and gas mutually heat each other across the shock front, therefore, dust re-radiation from high velocity clouds $\left(V_{\mathrm{s}}=500 \mathrm{~km} \mathrm{~s}^{-1}\right)$ peaks in the mid-IR (see Contini et al. 2002). Our results show that these clouds are rather dusty.

The data in the radio range do not follow exactly the slope corresponding to bremsstrahlung from a cool gas. Yet, they do not fit a power-law distribution (thin solid line), which could be explained by synchrotron radiation created by Fermi mechanism at the shock front. Figure 17 shows that both mechanisms contribute to the radio emission flux.

Finally, the thick solid lines in Fig. 17 represent the weighted sum of the single-cloud models for gas bremsstrahlung and dust reradiation, separately. The line is interrupted at frequencies lower than $10^{10} \mathrm{~Hz}$, because selfabsorption, which increases with wavelength, strongly reduces bremsstrahlung emission in the radio domain.

The model best fit to the data is obtained adopting different weights for different models. The weights account for the relative number of clouds corresponding to the model.

The ratio of the weights used to fit the data by the three models is $1: 0.2: 0.03$, the maximum weight corresponding to $V_{\mathrm{s}}=100 \mathrm{~km} \mathrm{~s}^{-1}$ and $D=0.17 \mathrm{pc}$ and the minimum to $V_{\mathrm{s}}=$ $500 \mathrm{~km} \mathrm{~s}^{-1}$. This confirms that velocities of $\sim 100 \mathrm{~km} \mathrm{~s}^{-1}$ and relatively small $D(<1 \mathrm{pc})$ dominate.

\section{Concluding remarks}

The central regions of the Seyfert-2 galaxy NGC 4388 have been observed by means of the integral field spectroscopy technique. The [O III] $\lambda 5007 / \mathrm{H} \beta$ map revealed part of the $\mathrm{S}-\mathrm{W}$ ionization cone, with $\mathrm{PA} \sim 200^{\circ}$ and aperture angle of $\sim 70^{\circ}$.

The analysis of the spectra within the cone shows the nonthermal active nature of the emission line regions, whose ionization degree remain high and almost constant even at large distances from the nucleus. With simple energy balance calculations we estimate the number of the ionizing photons produced by the engine and we demonstrate that even if the photoionization is the main physical process responsible for the excitation of the gas inside the cone, it cannot be considered the only one under way, since it cannot explain the observed high ionization far from the source.

We verify our hypothesis by applying to our spectroscopic data the SUMA code, which accounts for the combined effect of the photoionization from an external source and of the shocks. The results of the models, whose reliability is crosschecked by comparing them with the observed SED of the continuum, show that the ionization cone is characterized by a mixture of low density colliding clouds with large $(D \geq 1 \mathrm{pc})$ and small $(D<0.1 \mathrm{pc})$ geometrical thickness. Collisions, in fact, cause turbulence and $R-T$ and $K-H$ instabilities which lead to fragmentation of matter. In particular we are able to explain the high $[\mathrm{O} I I I] / \mathrm{H} \beta$ line ratios observed in the outer edges of the $\mathrm{S}-\mathrm{W}$ cone by the coupled effect of a low density gas in large clouds $(D \geq 1 \mathrm{pc})$.

The models show that the bulk of the emitting clouds within the S-W cone has shock velocity of $\sim 100 \mathrm{~km} \mathrm{~s}^{-1}$, definitively lower than those corresponding to the observed FWHM of the line profiles (200-700 $\mathrm{km} \mathrm{s}^{-1}$ ). Adopting the hypothesis that the extraplanar material in NGC 4388 may represent the tidal debris from a recent encounter with a small gas-rich galaxy (Pogge 1988; Veilleux et al. 1999b), we suggest that head-onback shocks accompany the outwards motion of the clouds, which result when an incoming gas catches up with preexistent gaseous clouds moving in the same direction with a different velocity.

To complete the analysis of the biconical structure of NGC 4388 we have modeled the emission line fluxes observed by YOSO2 in the VEELR of the N-E cone. We find that the physical conditions are in agreement with those found in the $\mathrm{S}-\mathrm{W}$ cone, taking into consideration dilution with distance from the AGN. The results lead to $\log F_{\mathrm{H}}=8.3-10.5$ $\left(U=2 \times 10^{-5}-5 \times 10^{-4}\right), n_{0}=30-100 \mathrm{~cm}^{-3}$, and $V_{\mathrm{s}} \sim 100-$ $300 \mathrm{~km} \mathrm{~s}^{-1}$ in the $\mathrm{S}-\mathrm{W}$ cone, and $\log F_{\mathrm{H}}=6.5-9.5\left(U=10^{-5}\right.$ $\left.1.5 \times 10^{-3}\right), n_{0}=2-10 \mathrm{~cm}^{-3}$, and $V_{\mathrm{s}}=30-150 \mathrm{~km} \mathrm{~s}^{-1}$ in the VEELR of the $\mathrm{N}-\mathrm{E}$ cone. We prefer to compare the radiation fluxes instead of the ionization parameter, because $U$ depends on the density of the gas in the edge of the cloud facing the radiation source. So, considering that the density decreases outward the galaxy, dilution is less prominent. The values of $U$ in the nuclear region, calculated by Petitjean \& Durret (1993), are much higher, $U=5 \times 10^{-3}-0.014$, indicating that different conditions characterize the gas in this region.

Concluding, densities, shock velocities, and the magnetic field are lower in the VEELR, suggesting that beyond the edges of the VEELR the emitting clouds are merging with the ISM. 
Acknowledgements. We are very grateful to the referee, D. Alloin, for precious comments which highly improved the presentation of the paper. M. Contini is grateful to the Astrophysikalisches Institut Potsdam and the Department of Astronomy Padova for warm hospitality. We are grateful to Prof. V. Afanasiev and Dr. S. Dodonov of the Special Astrophysical Observatory (Russia) for their decisive contribution in observations and useful discussions about data reduction and analysis. This research has made use of the NASA/IPAC Extragalactic Database (NED) which is operated by the Jet Propulsion Laboratory, California Institute of Technology, under contract with the National Aeronautics and Space Administration.

\section{References}

Allen, C. W. 1973, Astrophysical Quantities, 3rd ed. (Athlone Press) Becker, R. M., White, R. L., \& Edwards, A. L. 1991, ApJS, 75, 1

Bonatto, C., Bica, E., \& Alloin, D. 1989, A\&A, 226, 23

Boroson, T. A., Strom, K. M., \& Strom, S. E. 1983, ApJ, 274, 39

Colina, L. 1992, ApJ, 386, 59

Contini, M., \& Viegas, S. M. 2000, ApJ, 535, 721

Contini, M., \& Viegas, S. M. 2001, ApJS, 132, 211

Contini, M., Radovich, M., Rafanelli, P., \& Richter, M. G. 2002, ApJ, 572,124

de Vaucouleurs, A., \& Longo, G. 1988, Catalogue of Visual and Infrared Photometry of Galaxies from $0.5 \mu \mathrm{m}$ to $10 \mu \mathrm{m}$ (1961-1985) (Austin: University of Texas)

de Vaucouleurs, G., de Vaucouleurs, A., Corwin, J. R., et al. 1991, Third Reference Catalogue of Bright Galaxies (New York: Springer-Verlag)

Douglas, J. N., Bash, F. H., Bozyan, A., Torrence, G. W., \& Wolfe, C. 1996, AJ, 111, 1945

Dressel, L. L., \& Condon, J. J. 1978, ApJS, 36, 53

Fabbiano, G., Kim, D.-W., \& Trinchieri, G. 1992, ApJS, 80, 531

Falcke, H., Wilson, A. S., \& Simpson, C. 1998, ApJ, 502, 199

Ferland, J. G., \& Osterbrock, D. E. 1986, ApJ, 300, 658

Gregory, P. C., \& Condon, J. J. 1991, ApJS, 75, 1011
Halderson, E. L., Moran, E. C., Filippenko, A. V., \& Ho, L. C. 2001, AJ, 122, 637

Ho, L. C., Filippenko, A. V., \& Sargent, W. L. W. 1993, ApJ, 417, 63

Hummel, E., \& Saikia, D. 1991, A\&A, 249, 43

Kennicutt, R. C. 1998, ARA\&A, 36, 189

Kinney, A. L., Antonucci, R. R. J., Ward, M. J., Wilson, A. S., \& Whittle, M. 1991, ApJ, 377, 100

Kinney, A. L., Bohlin, R. C., Calzetti, D., Panagia, N., \& Wyse, R. F. G. 1993, ApJS, 86, 5

Matt, G., Piro, L., Antonelli, L. A., et al. 1994, A\&A, 292, L13

Miller, J. S., \& Mathews, W. G. 1972, ApJ, 172, 593

Moshir, M., Kopan, G., Conrow, T., et al. 1990, IRAS Faint Source Catalogue, version 2.0

Mould, J., Aaronson, M., \& Huchra, J. 1980, ApJ, 238, 458

Mulchaey, J. S., Koratkar, A., Ward, M. J., et al. 1994, ApJ, 436, 586

Petitjean, P., \& Durret, F. 1993, A\&A, 277, 365

Pogge, R. W. 1988, ApJ, 332, 702

Pogge, R. W. 1989, ApJ, 345, 730

Rafanelli, P., Violato, M., \& Baruffolo, A. 1995, AJ, 109, 1546

Scoville, N. Z., Becklin, E. E., Young, J. S., \& Capps, R. W. 1983, ApJ, 271, 512

Soifer, B. T., Boehmer, L., Neugebauer, G., \& Sanders, D. B. 1989, AJ, 98, 766

Spinoglio, L., Malkan, M. A., Rush, B., Carrasco, L., \& Recillas-Cruz, E. 1995, ApJ, 453, 616

Urry, C. M., \& Padovani, P. 1995, PASP, 107, 803

Veilleux, S., \& Osterbrock, D. M. 1987, ApJS, 63, 295

Veilleux, S., Bland-Hawthorn, J., \& Cecil, G. 1999a, AJ, 118, 2122

Veilleux, S., Bland-Hawthorn, J., Cecil, G., Tully, R. B., \& Miller, S. T. 1999b, ApJ, 520, 111

Viegas, S. M., \& Contini, M. 1994, ApJ, 428, 113

Yoshida, M., Yagi, M., Okamura, S., et al. 2002, ApJ, 567, 118

Zwicky, F., Herzog, E., \& Wild, P. 1961, Catalogue of Galaxies and of Clusters of Galaxies, vol. 1 (Pasadena: California Institute of Technology) 